\title{
Effects of laparoscopic and traditional open surgery on the levels of IL-6, TNF- $\alpha$, and Gal-3 in patients with thyroid cancer
}

\author{
Li He ${ }^{1,2 \#}$, Fangzhen Qing ${ }^{2,3 \#}$, Maode $\mathrm{Li}^{2,4}$, Daitian Lan ${ }^{2,4}$
}

${ }^{1}$ Department of Thyroid and Breast Surgery, Sichuan Provincial People's Hospital (East Hospital), University of Electronic Science and Technology of China, Chengdu, China; ${ }^{2}$ Chinese Academy of Sciences Sichuan Translational Medicine Research Hospital, Chengdu, China; ${ }^{3}$ Department of Stomatology, Sichuan Provincial People's Hospital (East Hospital), University of Electronic Science and Technology of China, Chengdu, China; ${ }^{4}$ Department of Hepatobiliary and Pancreatic Surgery, Sichuan Provincial People's Hospital (East Hospital), University of Electronic Science and Technology of China, Chengdu, China

Contributions: (I) Conception and design: M Li, L He, F Qing; (II) Administrative support: M Li; (III) Provision of study materials or patients: L He, F Qing; (IV) Collection and assembly of data: L He, F Qing; (V) Data analysis and interpretation: D Lan; (VI) Manuscript writing: All authors; (VII) Final approval of manuscript: All authors.

\#These authors contributed equally to this work and are co-first authors.

Correspondence to: Maode Li; Daitian Lan. Department of Hepatobiliary and Pancreatic Surgery, No. 585, Hong He North Road, Long Quan Yi District, Chengdu 610100, China. Email: kxian88806@sina.com; daitian0107@163.com.

Background: Traditional open surgery and laparoscopic surgery are common treatments for thyroid cancer patients, this paper aims to explore their effects on the levels of interleukin-6 (IL-6), tumor necrosis factor- $\alpha$ (TNF- $\alpha$ ), and galectin-3 (Gal-3) in patients with thyroid cancer.

Methods: The clinical data of patients with thyroid cancer who received surgery in our hospital from September 2017 to February 2020 were collected. In total, 106 cases that met the inclusion and exclusion criteria were included. The patients were then allocated into two groups according to the surgery received, including a study group (56 cases treated with endoscopy) and a basic group (50 cases treated with traditional open surgery). Rehabilitation indicators and inflammatory cytokines were compared between the two groups. Results: There was no significant difference in the number of intraoperative lymph node dissections ( $\mathrm{P}>0.05)$, postoperative complication rate $(16.08 \%$ vs. $20.00 \%, \mathrm{P}>0.05)$, and 6-month rate of recurrence or metastasis $(\mathrm{P}>0.05)$ between the two groups. Compared to the basic group, the operation time of the study group was longer, while the amount of intraoperative blood loss, $24 \mathrm{~h}$ drainage of the catheter and the length of hospital stay were significantly lower in the study group $(\mathrm{P}<0.05)$. The pain scores of the study group at 24 and $48 \mathrm{~h}$ after surgery were significantly lower than those of the basic group $(\mathrm{P}<0.05)$. The levels of IL-6, TNF- $\alpha$, Gal-3, and other inflammatory factors in the two groups increased on the first day postoperatively, however the levels of these factors in the study group were lower than those in the basic group $(\mathrm{P}<0.05)$. Finally, the postoperative cosmetic satisfaction rate of the study group (94.64\%) was higher than that of the basic group $(86.00 \%)$, and the difference was statistically significant $(\mathrm{P}<0.05)$.

Conclusions: The use of laparoscopic treatment can reduce the amount of intraoperative blood loss in patients with thyroid cancer, effectively reduce the degree of postoperative pain, and inhibit postoperative inflammation in the patient to a certain extent. Moreover, laparoscopic treatment can increase postoperative cosmetic satisfaction, reduce the occurrence of postoperative complications and recurrence rate, and improve the patient's prognosis.

Keywords: Laparoscopic surgery; traditional open surgery; thyroid cancer; interleukin-6 (IL-6); tumor necrosis factor- $\alpha(\mathrm{TNF}-\alpha)$; galectin-3 (Gal-3)

Submitted Jan 13, 2021. Accepted for publication Mar 02, 2021.

doi: $10.21037 /$ gs-21-60

View this article at: http://dx.doi.org/10.21037/gs-21-60 


\section{Introduction}

The thyroid gland is mainly composed of follicular epithelial cells and para-follicular epithelial cells, the uncontrolled growth of which may lead to the occurrence of thyroid cancer. Thyroid cancer is a common type of malignant tumor in the head and neck. The main clinical manifestations of thyroid cancer include a lump in the neck with swelling and pain, difficulty when swallowing and breathing, and frequent coughing, etc. (1). According to different cell origins, thyroid cancer can be divided into differentiated thyroid cancer (DTC), thyroid-like cancer, undifferentiated cancer, and other rare types, among which DTC is the most common type, accounting for more than $90 \%$ (2). This cancer is common in women; the incidence of thyroid cancer in women in China is approximately three times that of men.

Surgical resection is the main treatment for most thyroid cancers. Traditional open surgery and laparoscopic surgery are common treatments for thyroid cancer patients; both are widely used in clinical settings and both have their advantages, making the choice of surgical method controversial $(3,4)$. Galectin-3 (Gal-3) was characterized as a sensitive indicator for both differentiated and undifferentiated thyroid cancers (5), which could possibly improve the management of patients by reducing unnecessary thyroid surgery. This study explores the effects of laparoscopic and traditional open surgery on the levels of interleukin-6 (IL-6), tumor necrosis factor- $\alpha$ (TNF- $\alpha$ ), and Gal-3 in patients with thyroid cancer and aims to provide a reference for clinical treatment. We present the following article in accordance with the STROBE reporting checklist (available at http://dx.doi.org/10.21037/gs-21-60).

\section{Methods}

\section{General information}

The clinical data of patients with thyroid cancer who required surgery and were admitted to our hospital from September 2017 to February 2020 were collected. The inclusion criteria were as follows: (I) all patients that met the diagnostic criteria of the "Guidelines for the Diagnosis and Treatment of Thyroid Cancer (2015 Edition)" (6) and were confirmed by preoperative biopsy; (II) the tumor diameter did not exceed $3 \mathrm{~cm}$; (III) patients with no history of surgery or trauma in a relevant organ; (IV) the tumor was single and there was no cervical lymph node metastasis; and (V) the information was complete, and the patient voluntarily signed the informed consent. The exclusion criteria were as follows: (I) patients with serious diseases in vital organs; (II) patients who were intolerant to surgery and anesthesia; (III) patients with other malignant tumors and systemic infectious diseases; (IV) patients with abnormal coagulation function; and $(\mathrm{V})$ patients with abnormal mental state or poor coordination.

In total, this study included 106 patients who met the inclusion and exclusion criteria. The patients were allocated into two groups according to the surgery received: a study group (56 cases, receiving laparoscopic thyroidectomy) and a basic group (50 cases, receiving open surgery). In the study group, there were 16 males and 40 females, aged between 22 and 43 years, with an average of $30.28 \pm 3.16$ years. The patients in this group had tumors with a diameter ranging from 1 to $3 \mathrm{~cm}$ (average diameter, $1.25 \pm 0.97 \mathrm{~cm}$ ). Based on the pathological types, there were 38 cases with papillary thyroid carcinoma and 18 cases with thyroid follicular carcinoma, and according to the affected side, 30 cases were on the left side and 26 cases were on the right side.

Patients in the basic group were aged between 23 and 42 years, with an average age of $30.56 \pm 3.27$ years, and a tumor diameter ranging from 1 to $3 \mathrm{~cm}$ (average diameter, $1.16 \pm 1.02 \mathrm{~cm})$. According to the pathological type, patients in the basic group were divided into papillary thyroid carcinoma (35 cases) and thyroid follicular carcinoma (15 cases), and according to the affected side, 27 cases were on the left side and 23 cases were on the right side. There were no significant differences between the two groups in terms of clinical data such as gender, age, tumor diameter, pathological type, etc. $(P>0.05)$.

All procedures performed in this study involving human participants were in accordance with the Declaration of Helsinki (as revised in 2013). The study was approved by the ethics committee of Sichuan Provincial People's Hospital. Written informed consent was obtained from the patients.

\section{Surgery}

Both groups of patients completed preoperative examinations, including lung computed tomography (CT), neck radiography, electrocardiogram, laryngoscopy, thyroid function test, as well as routine blood and biochemical tests, and were fully prepared for the operation.

Traditional open surgery treatment was used for patients in the basic group (7). Specifically, the patient lay in the supine position with the shoulders raised to hyperextend 

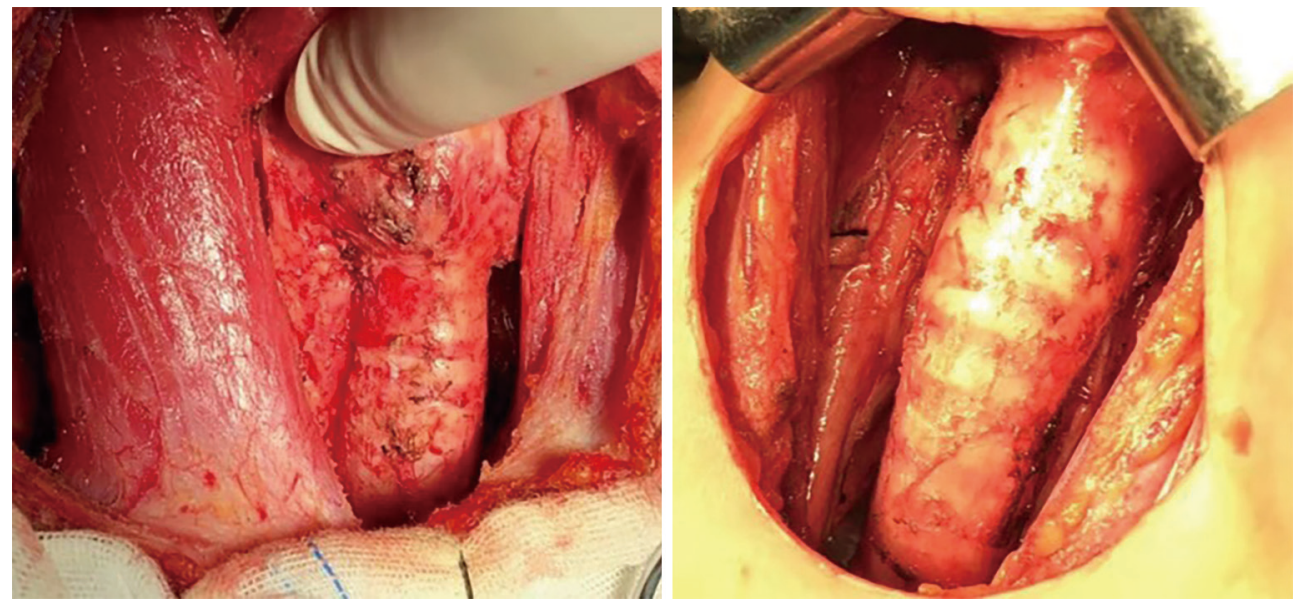

Figure 1 Pictures showing open surgery for thyroid cancer.

the neck. Tracheal intubation was performed for general anesthesia, and the surgical site was then routinely disinfected and draped. A 6-cm arc-shaped incision was then made at the position approximately $3 \mathrm{~cm}$ to the suprasternal fossa, and the muscle tissue covering the thyroid lobe was then separated layer by layer, so that the thyroid lobe was fully exposed. Partial or total resection of the affected side of the thyroid gland was performed, the central lymph nodes were cleaned, and a drainage tube was then placed in the wound. The incision was subsequently sutured, and the operation was completed (Figure 1).

Laparoscopic thyroidectomy was used for patients in the study group (8). The patient lay in the supine position with the shoulders raised to hyperextend the neck, and the neck was completely exposed to the field of vision. Tracheal intubation was then performed for general anesthesia, and the surgical site was routinely disinfected and draped. Surgical path: breast milk path. Diluted epinephrine was injected into the superficial fascia of the anterior sternum to prevent bleeding. A $10-\mathrm{mm}$ incision was made to the patient's anterior midline of the flat nipple, and the trocar and cavity lens were subsequently inserted. The incision served as the observation point, and carbon dioxide $\left(\mathrm{CO}_{2}\right)$ was filled to maintain the operating space. Next, 10 and $7 \mathrm{~mm}$ arc incisions were made $5 \mathrm{~mm}$ to the upper edge of the areola on both sides, reaching the deep superficial fascia layer. These two incisions served as operating points, and the trocar and cavity lens were placed for surgery. An ultrasonic knife was used to penetrate the deep superficial fascia, and to incise the subcutaneous loose connective tissue to expose the suprasternal fossa and the inner edge of the sternocleidomastoid muscle, and continued to the level of the lower edge of the thyroid cartilage. Subsequently, the ultrasonic knife was then used to separate the anterior neck muscle layer from the neck white line to the affected location and to cut the envelope covering the outer layer of the thyroid. Once the recurrent laryngeal nerve and parathyroid glands were exposed, they were taken care of, and the thyroidectomy was then applied to the affected side. The lymph nodes in the central area were swept and the surgical site was continuously washed with distilled water. After the sternocleidomastoid muscle was thoroughly cleaned, a drainage tube was placed on the upper edge of the areola, and the wound was sutured layer by layer (Figure 2).

\section{Observation indicators}

\section{Surgery-related indicators}

The operation time, intraoperative blood loss, $24 \mathrm{~h}$ drainage of the catheter, number of lymph node dissections, and length of hospital stay were recorded by the responsible nurse.

\section{Pain score at different periods after the operation}

The main complaint level of pain was used for the grading of pain (9). Specifically, 0-3 points signified that the patient felt pain but could tolerate it, and the pain did not interfere with normal life or sleep; 4-7 points meant that the patient had obvious pain that was unbearable, required medication for pain relief, and sleep was disturbed; $8-10$ points denoted that the patient had severe pain and required medication for analgesia, the pain seriously interfered with sleep, and 

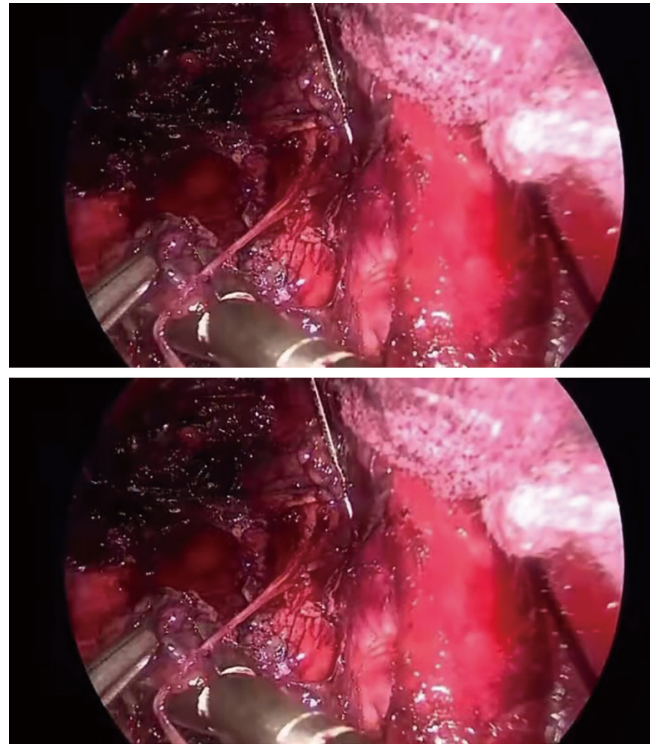

Figure 2 Pictures of the treatment of thyroid cancer with laparoscopic thyroidectomy.

passive posture may appear. Using this scoring system, the patients were evaluated at 24,48 , and $96 \mathrm{~h}$ after surgery.

\section{Inflammatory factors}

The levels of IL-6 (JL14113), TNF- $\alpha$ (JL10208), and Gal-3 (JL18302), and other inflammatory indicators were measured $96 \mathrm{~h}$ before and $36 \mathrm{~h}$ after surgery. For all of these measurements, the double antibody sandwich enzymelinked immunosorbent assay (ELISA) method was used. All kits were provided by Shanghai Jianglai Biotechnology Co., Ltd. (Shanghai, China), and the relevant operations were carried out in strict accordance with the manufacturer's instructions.

\section{Postoperative complications and recurrence}

Postoperative complications, including difficulty drinking water, choking, hoarseness, numbness of the limbs, and temporary hypocalcemia, were determined after the operation. Total incidence $=$ sum of all complications $/$ total number of cases $\times 100 \%$. After discharge from the hospital, all patients were followed up regularly for 6 months. CT was used to check for recurrence, and the recurrence rate was calculated accordingly.

\section{Cosmetic satisfaction}

Cosmetic satisfaction, including satisfaction, general satisfaction, and dissatisfaction (10), was also determined. Specifically, satisfaction meant that the incision healed with a small scar, and the incision had no depression or bulge. General satisfaction signified that the patient's incision healed well, the scar was small, and the incision had slight depression or bulge. Dissatisfaction denoted that the patient's incision had not healed well, the scar was large, and the incision had obvious depressions and bulges. Total satisfaction $=$ (number of satisfactory cases + number of general satisfaction cases)/total number of cases $\times 100 \%$. The patients' evaluation of satisfaction with the operation were collected at the third month of follow-up after discharge.

\section{Statistical analysis}

The data in this study were analyzed by SPSS 22.0 software (IBM, US). Count data were expressed as $\mathrm{n}(\%)$, and the F/ $t$-test was used. Measurement data were expressed by the mean \pm standard deviation $\left(\bar{x}_{ \pm}\right)$, and the chi-squared $\left(\chi^{2}\right)$ test was used for the comparison of the measurement data. Differences were considered statistically significant when $\mathrm{P}<0.05$.

\section{Results}

\section{Comparison of the surgical indicators between the two groups}

There was no significant difference in the number of lymph node dissections between the two groups $(\mathrm{P}>0.05)$. The operation time of the study group was longer than that of the basic group, while the amount of blood loss, $24 \mathrm{~h}$ drainage of the catheter, and the length of hospital stay were significantly lower in the study group compared to the basic group, and the difference was statistically significant $(\mathrm{P}<0.05$, Table 1).

\section{Comparison of the pain scores between the two groups at different periods postoperatively}

There were no differences in the pain scores between the two groups at $96 \mathrm{~h}$ postoperatively $(\mathrm{P}>0.05)$. However, with time, the pain scores of the two groups gradually decreased, and the pain scores of the study group at 24 and $48 \mathrm{~h}$ after surgery were significantly lower than those of the basic group, $(\mathrm{P}<0.05$, Table 2). 
Table 1 Comparison of various surgical indicators between the two groups $(\bar{x} \pm s)$

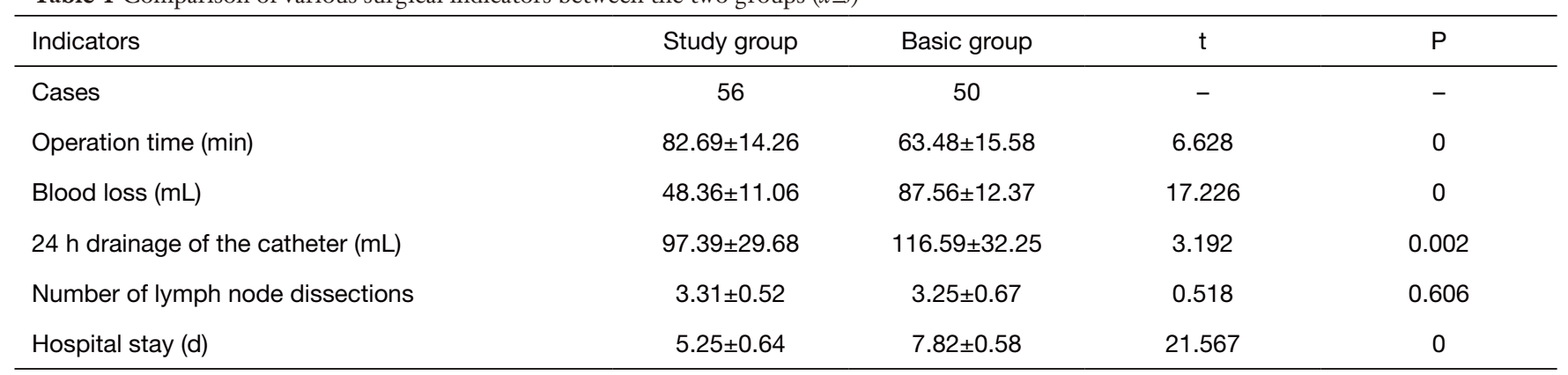

Table 2 Comparison of pain scores ( $\bar{x} \pm s$, points) between the two groups at different periods after surgery

\begin{tabular}{lcccccc}
\hline Groups & Cases & 24 h postoperatively & 48 h postoperatively & 96 h postoperatively & $F$ & $P$ \\
\hline Study group & 56 & $5.23 \pm 1.10$ & $4.02 \pm 1.25$ & $3.20 \pm 0.73$ & 53.01 & 0.000 \\
Basic group & 50 & $6.87 \pm 1.56$ & $5.42 \pm 1.32$ & $3.05 \pm 0.61$ & 137.36 \\
$\mathrm{t}$ & - & 6.185 & 5.606 & 1.140 & - \\
$\mathrm{P}$ & - & 0.000 & 0.000 & 0.257 & - \\
\hline
\end{tabular}

Table 3 Comparison of serum inflammatory factor levels between the two groups $(\bar{x} \pm s)$

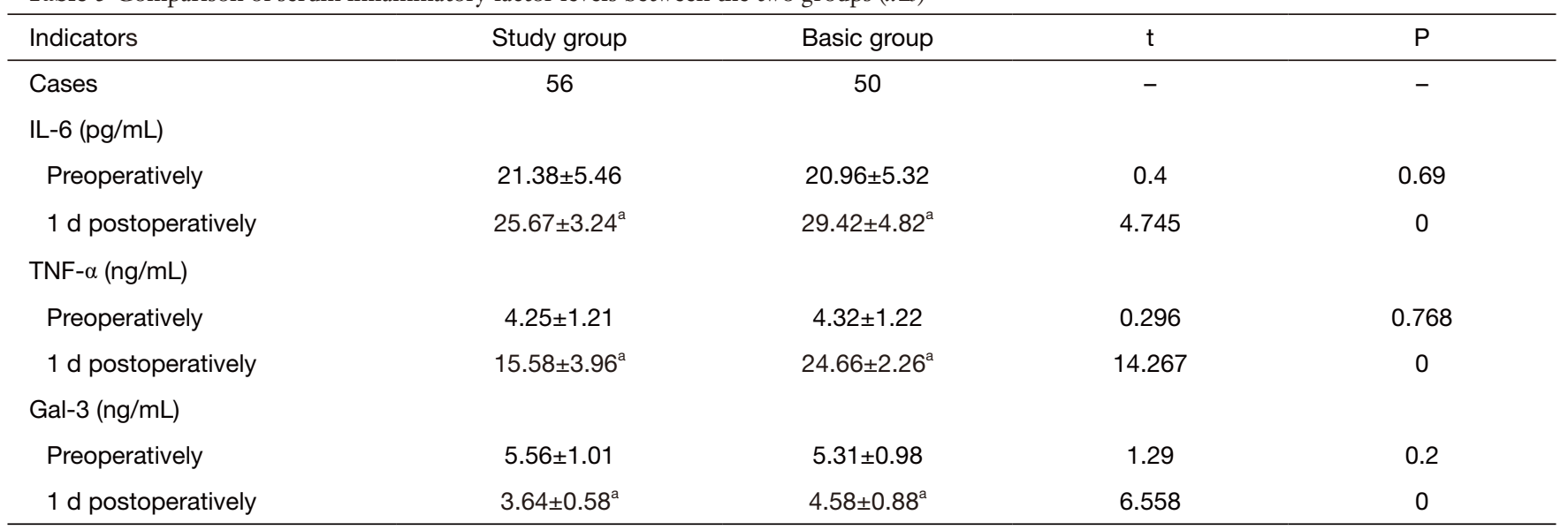

${ }^{\mathrm{a}}, \mathrm{P}<0.05$ versus preoperatively.

\section{Comparison of serum inflammatory factor levels between the two groups}

There were no significant differences in the levels of IL-6, TNF- $\alpha$, Gal-3, and other inflammatory factors between the two groups preoperatively $(\mathrm{P}>0.05)$. However, 1 day after the operation, the levels of inflammatory factors including IL-6, TNF- $\alpha$, Gal-3, etc. were increased compared with their preoperative levels. The increased levels of the above indicators were significantly lower in the study group compared to those in the basic group $(\mathrm{P}<0.05$, Table 3).

\section{Comparison of postoperative complications and recurrence between the two groups}

As shown in Table 4, the incidence of postoperative complications in the study group (16.08\%) and the basic group $(20.00 \%)$ was not statistically different $(P>0.05)$. Furthermore, there was no recurrence or metastasis in 
Table 4 Comparison of postoperative complications between the two groups [n (\%)]

\begin{tabular}{lcccc}
\hline Indicators & Study group & Basic group & $\chi^{2}$ & $P$ \\
\hline Cases & 56 & 50 & - & - \\
Limb numbness & $1(1.79)$ & $3(6.00)$ & 0.542 & 0.462 \\
Coughing during & $1(1.79)$ & $0(0.00)$ & & \\
drinking & & & & \\
Hoarse voice & $2(3.57)$ & $1(2.00)$ & & \\
Hypocalcemia & $5(8.93)$ & $6(12.00)$ & & \\
Total incidence (\%) & 16.08 & 20 & & \\
\hline
\end{tabular}

the two groups of patients during the 6 months follow-up period after discharge.

\section{Comparison of postoperative cosmetic satisfaction rates between the two groups}

As shown in Table 5, the postoperative cosmetic satisfaction rate of the study group (94.64\%) was higher than that of the basic group $(86.00 \%)$, and the difference was statistically significant $(\mathrm{P}<0.05)$.

\section{Discussion}

Thyroid cancer is a malignant tumor. An enlarged thyroid or nodules are common symptoms, and manifest as hard nodules of the thyroid, which can move up and down when swallowing. Radiation exposure, viral infection, or family inheritance can all be the triggers for DTC, especially for people with a history of radioactive exposure, and the risk of disease is significantly increased (11). DTC accounts for more than $90 \%$ of thyroid cancers. DTC has a relatively low malignancy, and thus its treatment efficacy is more significant; however, cancer cells can often metastasize to the neck, which can aggravate the condition and lead to poor prognostic survival (12). Thyroid cancer resection combined with lymph node dissection can effectively inhibit the development of the patient's disease by removing the tumor tissue, and thereby prolong the patient's disease-free survival.

Laparoscopic surgery and traditional open surgery are widely used in the treatment of thyroid cancer $(13,14)$. However, some studies have found that open thyroidectomy may cause damage to the tissues and nerves around the thyroid. If the recurrent laryngeal nerve is injured, the patient will have persistent hoarseness and difficulty
Table 5 Comparison of postoperative cosmetic satisfaction rate between the two groups [n (\%)]

\begin{tabular}{lcccc}
\hline Indicators & Study group & Basic group & $\chi^{2}$ & $\mathrm{P}$ \\
\hline Cases & 56 & 50 & - & - \\
Satisfaction & $25(44.64)$ & $17(34.00)$ & 4.711 & 0.03 \\
General satisfaction & $28(50.00)$ & $26(52.00)$ & & \\
Dissatisfaction & $3(5.36)$ & $7(14.00)$ & & \\
Satisfaction rate $(\%)$ & 94.64 & 86 & & \\
\hline
\end{tabular}

when eating, in addition to other complications, and their long-term recurrence and tumor progression rates remain high (15). It has been found that total laparoscopic thyroidectomy can reduce surgical wounds and improve the cosmetic appearance of the wound following an incision. The results of this study showed that the postoperative cosmetic satisfaction rate $(94.64 \%)$ of the study group was higher than that of the basic group $(86.00 \%)$, and the difference was statistically significant $(\mathrm{P}<0.05)$, which is consistent with other reports.

Furthermore, the trauma caused by full laparoscopic surgery is small, the anatomical position of nerves and other tissues can be clearly observed during laparoscopic surgery, and the protection of the recurrent laryngeal nerve can also be improved under the pressure of gas (16). Studies have demonstrated that laparoscopic thyroidectomy has a higher rate of complete resection of local thyroid gland tissue, and the dissection of the lymph node area is also relatively complete. The results of this study showed that the amount of intraoperative blood loss, pain at 24 and $48 \mathrm{~h}$ postoperatively, and the length of hospital stay were significantly lower in the study group compared to the basic group; however, the overall operation time was longer $(\mathrm{P}<0.05)$. These findings suggest that the small wounds of the laparoscopic surgery can effectively reduce the risk of bleeding from the wound, thereby reducing the degree of postoperative pain in patients. However, due to the difficulty of laparoscopic surgery, the operation time will inevitably be longer. This requires that the surgeon practice the operation under the laparoscopy to improve proficiency in order to shorten the operation time and reduce the risk.

Since surgery will cause unavoidable trauma to the patient's body, the inflammatory reaction that occurs at the wound site will cause transient high expression levels of inflammatory factors, such as TNF- $\alpha$, Gal-3, and IL-6, which are effective indicators to assess the degree of the postoperative inflammatory response in patients (17). IL-6 
is a pleiotropic cytokine with a wide range of functions. It participates in the immune response, acute-phase response, and hematopoietic function, and also plays an important role in acute inflammation, such as internal and external trauma, surgery, infection, etc. In these cases, it can be rapidly generated, and thus serves as an indicator for early inflammation in the body (18). TNF- $\alpha$ is a multidirectional pro-inflammatory factor, which participates in the occurrence of the local inflammatory reaction, and is also often used as an index to clinically detect the degree of the inflammatory response in the body (19). Gal-3 can promote angiogenesis, tumor occurrence, and metastasis, and is also a powerful pro-inflammatory factor (20). In this study, by measuring the levels of TNF- $\alpha$, Gal-3, and IL-6 in patients with thyroid cancer before and after surgery, we observed that the two groups of patients showed an increase in the abovementioned inflammatory factors on the first day after surgery. This suggests that the patients had an acute postoperative inflammatory response, however the degree of postoperative inflammatory factor increase in the study group was significantly lower than that of the basic group, indicating that laparoscopic surgery can effectively reduce the patient's postoperative inflammatory response. A small number of patients in both groups had postoperative complications, but they were able to relieve themselves or improved following treatment. The absence of recurrence during the 6-month postoperative follow-up period indicates the high safety of laparoscopic surgery (21).

In summary, laparoscopic surgery generates smaller wounds, high cosmetic satisfaction, and can reduce intraoperative blood loss and postoperative pain in patients with thyroid cancer. It can also improve postoperative recovery and shorten hospitalization time. With its high safety, laparoscopic surgery can also effectively suppress the degree of postoperative inflammation in patients.

\section{Acknowledgments}

Funding: None.

\section{Footnote}

Reporting Checklist: The authors have completed the STROBE reporting checklist. Available at http://dx.doi. org/10.21037/gs-21-60

Data Sharing Statement: Available at http://dx.doi. org/10.21037/gs-21-60
Conflicts of Interest: All authors have completed the ICMJE uniform disclosure form (available at http://dx.doi. org/10.21037/gs-21-60). The authors have no conflicts of interest to declare.

Ethical Statement: The authors are accountable for all aspects of the work in ensuring that questions related to the accuracy or integrity of any part of the work are appropriately investigated and resolved. All procedures performed in this study involving human participants were in accordance with the Declaration of Helsinki (as revised in 2013). The study was approved by the ethics committee of Sichuan Provincial People's Hospital. Written informed consent was obtained from the patients.

Open Access Statement: This is an Open Access article distributed in accordance with the Creative Commons Attribution-NonCommercial-NoDerivs 4.0 International License (CC BY-NC-ND 4.0), which permits the noncommercial replication and distribution of the article with the strict proviso that no changes or edits are made and the original work is properly cited (including links to both the formal publication through the relevant DOI and the license). See: https://creativecommons.org/licenses/by-nc-nd/4.0/.

\section{References}

1. Spinelli C, Strambi S, Bakkar S, et al. Surgical Management of Diffuse Sclerosing Variant of Papillary Thyroid Carcinoma. Experience in 25 Patients. World J Surg 2020;44:155-62.

2. Maroof H, Islam F, Ariana A, et al. The roles of microRNA-34b-5p in angiogenesis of thyroid carcinoma. Endocrine 2017;58:153-66.

3. Gigliotti CL, Ferrara B, Occhipinti S, et al. Enhanced cytotoxic effect of camptothecin nanosponges in anaplastic thyroid cancer cells in vitro and in vivo on orthotopic xenograft tumors. Drug Deliv 2017;24:670-80.

4. Liu XW, Li Q, Chen F, et al. Efficacy of three-dimensional laparoscopic total thyroidectomy combined with central lymph node dissection for thyroid cancer and its effect on inflammatory responses of the patients. Nan Fang Yi Ke Da Xue Xue Bao 2017;37:842-6.

5. De Rose F, Braeuer M, Braesch-Andersen S, et al. Galectin-3 Targeting in Thyroid Orthotopic Tumors Opens New Ways to Characterize Thyroid Cancer. J Nucl Med 2019;60:770-6.

6. Pishkari S, Paryan M, Hashemi M, et al. The role of 
microRNAs in different types of thyroid carcinoma: a comprehensive analysis to find new miRNA supplementary therapies. J Endocrinol Invest 2018;41:269-83.

7. Nickel B, Semsarian C, Moynihan R, et al. Public perceptions of changing the terminology for low-risk thyroid cancer: a qualitative focus group study. BMJ Open 2019;9:e025820.

8. Cho YJ, Kim DY, Park EC, et al. Thyroid fine-needle aspiration biopsy positively correlates with increased diagnosis of thyroid cancer in South Korean patients. BMC Cancer 2017;17:114.

9. Tam AA, Ozdemir D, Aydın C, et al. Association between preoperative thyrotrophin and clinicopathological and aggressive features of papillary thyroid cancer. Endocrine 2018;59:565-72.

10. Metere A, Frezzotti F, Graves CE, et al. A possible role for selenoprotein glutathione peroxidase (GPx1) and thioredoxin reductases (TrxR1) in thyroid cancer: our experience in thyroid surgery. Cancer Cell Int 2018;18:7.

11. Moore MD, Postma E, Gray KD, et al. Less is More: The Impact of Multidisciplinary Thyroid Conference on the Treatment of Well-Differentiated Thyroid Carcinoma. World J Surg 2018;42:343-9.

12. Debnam JM, Guha-Thakurta N, Sun J, et al. Distinguishing Recurrent Thyroid Cancer from Residual Nonmalignant Thyroid Tissue Using Multiphasic Multidetector CT. AJNR Am J Neuroradiol 2020;41:844-51.

13. Choi MH, Kim KA, Hwang SS, et al. CT-quantified muscle and fat change in patients after surgery or endoscopic resection for early gastric cancer and its impact on long-term outcomes. Medicine (Baltimore) 2018;97:e13878.

14. Bellantone R, Raffaelli M, De Crea C, et al. VideoAssisted Thyroidectomy for Papillary Thyroid Carcinoma:

Cite this article as: He L, Qing F, Li M, Lan D. Effects of laparoscopic and traditional open surgery on the levels of IL-6, TNF- $\alpha$, and Gal-3 in patients with thyroid cancer. Gland Surg 2021;10(3):1085-1092. doi: 10.21037/gs-21-60
Oncologic Outcome in Patients with Follow-Up $\geq 10$ Years. World J Surg 2018;42:402-8.

15. Prete FP, Ria R, Gurrado A, et al. Response to letter: "Effect of thyroidectomy on circulating angiogenic cytokines in papillary thyroid carcinoma and benign goiter: Potential for new biomarkers?". Surgery 2020. [Epub ahead of print]. doi: 10.1016/j.surg.2020.10.030.

16. Inabnet WB, 3rd, Fernandez-Ranvier G, Suh H. Transoral Endoscopic Thyroidectomy-An Emerging Remote Access Technique for Thyroid Excision. JAMA Surg 2018;153:376-7.

17. Pereira-Prado V, Vigil-Bastitta G, Sánchez-Romero C, et al. Immunoexpression of galectin-3 and its potential relation to hypoxia-inducible factor- $1 \alpha$ in ameloblastomas. Biotech Histochem 2020. [Epub ahead of print]. doi: 10.1080/10520295.2020.1800819.

18. Insilla AC, Proietti A, Borrelli N, et al. TERT promoter mutations and their correlation with BRAF and RAS mutations in a consecutive cohort of 145 thyroid cancer cases. Oncol Lett 2018;15:2763-70.

19. Paouri E, Tzara O, Kartalou GI, et al. Peripheral Tumor Necrosis Factor-Alpha (TNF- $\alpha$ ) Modulates Amyloid Pathology by Regulating Blood-Derived Immune Cells and Glial Response in the Brain of AD/TNF Transgenic Mice. J Neurosci 2017;37:5155-71.

20. Ayroldi E, Petrillo MG, Marchetti MC, et al. Long glucocorticoid-induced leucine zipper regulates human thyroid cancer cell proliferation. Cell Death Dis 2018;9:305.

21. Kim SC, Kim JH, Won JK, et al. Asymptomatic intrathyroidal pyriform sinus fistula mimicking thyroid cancer: A case report and literature review. Medicine (Baltimore) 2018;97:e0488.

(English Language Editor: A. Kassem) 